\title{
The risk factors of portal vein thrombosis in patients with liver cirrhosis
}

\author{
YASIR FURKAN CAGIN ${ }^{1}$, YILMAZ BILGIC ${ }^{1}$, ILHAMI BERBER ${ }^{2}$, \\ OGUZHAN YILDIRIM ${ }^{1}$, MEHMET ALI ERDOGAN ${ }^{1}$, FEYZA FIRAT ${ }^{3}$, AHMET KADIR ARSLAN $^{4}$, \\ CEMIL COLAK $^{4}$, YUKSEL SECKIN ${ }^{1}$ and MURAT HARPUTLUOGLU ${ }^{1}$ \\ ${ }^{1}$ Department of Gastroenterology, Medical Faculty, Inonu University, 44280 Malatya; ${ }^{2}$ Clinic of Hematology, \\ Malatya Training and Education Hospital, 44330 Malatya; Departments of ${ }^{3}$ Internal Medicine and ${ }^{4}$ Biostatistics \\ and Medical Informatics, Medical Faculty, Inonu University, 44280 Malatya, Turkey
}

Received January 29, 2018; Accepted November 23, 2018

DOI: $10.3892 /$ etm.2019.7300

\begin{abstract}
This study was designed to identify and assess risk factors for portal vein thrombosis (PVT) in patients with cirrhosis. A total of 98 cirrhosis patients with PVT were identified and 101 cirrhosis patients without PVT were chosen as the control group in this retrospective study. Several variables were measured and the two groups PVT and non-PVT were compared statistically. PVT was identified in 98 patients $(10 \%)$. Significant differences in hematocrit, international normalized ratio, albumin, bilirubin and glucose were determined between the groups $(\mathrm{P}<0.05)$. Out of the thrombophilic risk factors in the patients with PVT factor V Leiden was identified in $8.8 \%$, prothrombin gene $6.6 \%$ and methylenetetrahydrofolate reductase $2.2 \%$. There was no difference in survival time between groups $(\mathrm{P}>0.05)$.
\end{abstract}

\section{Introduction}

Portal vein thrombosis (PVT) is a complication that frequently occurs during the course of liver cirrhosis, especially in advanced stages. Although it is considered a rare event, PVT has been increasingly identified due to the increased frequency of liver imaging techniques used in patients with cirrhosis $(1,2)$. The prevalence of PVT is $\sim 1 \%$ in the general population and investigators have demonstrated in different series that it develops in $5-20 \%$ of cirrhosis patients. However, there are differences in the reported prevalence of PVT (3-5).

Correspondence to: Dr Yasir Furkan Cagin, Department of Gastroenterology, Medical Faculty, Inonu University, 10 Malatya Elazı̆̆ Road, Battalgazi, 44280 Malatya, Turkey

E-mail: yafur@hotmail.com

Dr İlhami Berber, Clinic of Hematology, Malatya Training and Education Hospital, 4 Turgut Ozal Avenue, Yesilyurt, 44330 Malatya, Turkey

E-mail: drilhamiberber@gmail.com

Key words: cirrhosis, portal vein thrombosis, risk factors
There are various etiological factors that are considered to predispose liver cirrhosis patients to the formation of PVT, including inflammation, neoplasms, coagulation disorders, trauma and abdominal surgery. In a study of patients with cirrhosis, it was demonstrated that prothrombotic states were more frequent in patients with PVT compared with patients without PVT (6). While the mechanism of the occurrence of PVT in cirrhosis patients is not still clear, studies have demonstrated that there is a close association between PVT and advanced liver disease (7). In this regard, several risk factors have been proposed for this complication (8). The development of PVT is a multifactorial process. Frequently, more than one risk factor is identified, whereas a single factor is rarely identified in cirrhosis patients (9). Hepatic structural derangement secondary to a slowing of portal vein blood flow is primarily responsible for PVT; damage to the vessel wall and hypercoagulability serve important roles as well. Inherited and acquired thrombotic risk factors also serve a role (10). The presence of PVT in cirrhosis patients is very important, as it can lead to technical difficulties in liver transplantation and active variceal bleeding $(11,12)$.

The purpose of the study was to determine the clinical features of PVT in patients with liver cirrhosis attending a tertiary support hospital in Malatya, Turkey and to recognize the biochemical factors associated with PVT.

\section{Materials and methods}

Guidelines and data availability. Ethics committee approval is not required for retrospective studies in Inonu University Medical Faculty (Malatya, Turkey). From January 2009 to March 2015, overall 978 patients with liver cirrhosis (605 men, 373 women) were enrolled in the database of the Gastroenterology Unit of Turgut Ozal Medical Center, a tertiary care hospital in Malatya, Turkey.

Study selection. Liver cirrhosis was diagnosed if the patient had a positive liver biopsy on record, by imaging techniques or clinical signs including evidence of portal hypertension, ascites and encephalopathy. However, the seriousness of the liver disease was classified in accordance with Child-Pugh class 
A to $\mathrm{C}$ and model for end-stage liver disease (MELD) (13). The exclusion criteria for the patients were as follows: Liver tumor and other malignant tumors, liver transplantation, Budd-Chiari syndrome, splenectomy, non-liver disease complicated (contraceptive use, previous venous thrombosis, familial thrombophilia, abdominal surgery, abdominal trauma or sepsis or overt myeloproliferative disorders) by PVT and usage of anticoagulant or anti-platelet medications. Patients with hepatocellular carcinoma were eliminated by abdominal ultrasound, spiral abdominal computed tomography and $\alpha$-fetoprotein values. The number of individuals was determined based on power analysis given below and their characteristics are provided in Table I.

Creation of groups. A total of 98 cirrhosis patients with PVT were identified from the retrospective patient records. In order to recognize factors associated with PVT, 101 consecutive cirrhosis patients without PVT from 880 patients remaining were chosen randomly to make up the control group. The median ages were 56 years (range, 6-83) in the PVT group and 53 years (range, 16-87) in the non-PVT group. A total of two groups were formed: The patient (PVT) and control (non-PVT) groups. PVT was categorized as complete or partial if thrombus was concluded as nonexistent or if lessening of blood flow occurred in the main portal trunk, left and right branches of the portal vein, superior mesenteric vein and splenic vein; the existence of a portal cavernoma was appraised. The diagnosis of PVT was made by Doppler flow imaging, as is routine. When diagnosis of PVT was uncertain, it was verified by computed tomography or magnetic resonance imaging. In addition to imaging, patients who had not yet developed the characteristics of chronic PVT, including collateral circulation (cavernous portal transformation) or portal hypertension were deemed to have acute PVT. Acute PVT was exhibited in $40 \%$ of all patients with PVT. Patient clinical features and the prevalence of patient risk factors are listed in Tables I and II. The records of the cirrhosis patients were appraised to attain data regarding demographic characteristics (age, gender) and hematocrit (HTC), platelet (PLT), international normalized ratio (INR), albumin (ALB), bilirubin (BLB), aspartate aminotransferase (AST), alanine aminotransferase (ALT), sodium (Na), creatinine (CR), and glucose values obtained at baseline. The variables were measured and the groups were compared statistically.

All the databases were screened for thrombophilia panel including protein $\mathrm{C}, \mathrm{S}$, antithrombin III, fasting plasma homocysteine, the mutation of factor V Leiden (FVL), prothrombin gene $(\mathrm{PG})$ and the methylenetetrahydrofolate reductase (MTHFR) enzyme. Due to the limitations of a retrospective study, the thrombophilic risk factors in the control group had not been previously studied before the beginning of the present study. The aforementioned factors were measured and the groups were compared statistically with respect to the factors after the start of the present study. In addition, the medical guideline of the STrengthening the Reporting of OBservational studies in Epidemiology (STROBE) was completed, which evaluates the quality of observational studies.

Sample size. The minimum sample size required to detect a significant difference of HCT values between the groups should be at least 88 in each group, (176 in total), considering a type I error $(\alpha)$ of 0.05 , power $(1-\beta)$ of 0.8 , effect size of 0.43 and two-sided alternative hypothesis (H1). To increase the power of this study, 199 individuals (98 in PVT group; 101 in non-PVT group) were included in the present study.

Statistical analysis. Categorical variables were given as frequencies with percentages and continuous variables were presented as median (min-max) based on the distribution of variables. Normality was confirmed with the Shapiro-Wilk test. Pearson's chi-square test was used for categorical variables and the Mann-Whitney $U$ test was used to determine non-normally distributed variables. $\mathrm{P}<0.05$ was considered to indicate a statistically significant difference. Backward stepwise multiple logistic regression models were conducted to predict associations between the predictor variables (age, gender, etiology, HTC, PLT, INR, ALB, BLB, AST, ALT, Na, $\mathrm{CR}$, glucose, Child-Pugh class, MELD score and thrombophilia panel) and dependent variable (PVT/Non-PVT groups). The results of the predicted model were assessed with the Hosmer-Lemeshow test and Omnibus test of the model coefficient. Mortality rates were analyzed for all patients by the Kaplan-Meier method and statistical differences were defined by the log-rank test. Kaplan-Meier survival curves were formed from the survival time of patient according to being dead and alive. All analyses were conducted using IBM SPSS Statistics 23.0 software (IBM Corporation, Armonk, NY).

\section{Results}

Study and patient characteristics. This study consisted of 199 individuals: 98 (49.2\%) in the PVT group and $101(50.8 \%)$ in the non-PVT group. The descriptive statistics of the groups are provided in Table I.

Parameter analysis. Significant differences in HTC, INR, ALB, BLB and glucose were identified among the groups $(P<0.05)$. There were no significant differences in the other variables between the two groups $(\mathrm{P}>0.05)$. Among the patients with PVT, 13 (13.3\%) were in Child-Pugh class A, $37(37.8 \%)$ in class B and $48(48.9 \%)$ in class C. There were no significant differences in Child-Pugh classes among the groups. Among the thrombophilic risk factors in patients with PVT, FVL (12.2\%), PTHR 20210 (15.3\%) and MTHFR (16.3\%) were identified. Furthermore, a significant difference between the groups was identified $(\mathrm{P}<0.05)$. It was also revealed that the FVL mutation was more frequent in cirrhotic patients with PVT compared with control groups (Table II).

Statistical results. The results of backward stepwise multiple logistic regression modeling are presented in Table III. According to these results, age and HTC were significantly associated with PVT $(\mathrm{P}<0.05)$. The Hosmer-Lemeshow test indicated that the backward stepwise multiple logistic regression fit well to the data of interest ( $\mathrm{P}=0.568$; Table III). The Omnibus tests of model coefficients were statistically significant $(\mathrm{P}<0.001$; Table III).

The Kaplan-Meier analysis of patient survival time (years) was performed among groups and was demonstrated in Figs. 1 and 2. The survival time of the patients was compared and the different was not significant $(\mathrm{P}>0.05)$. 
Table I. The clinical features and the descriptive statistics of the groups.

\begin{tabular}{|c|c|c|c|}
\hline Factors & $\operatorname{PVT}(\mathrm{n}=98)$ & Non-PVT $(\mathrm{n}=101)$ & P-value \\
\hline Age $^{a}$ & $56(6-83)$ & $53(16-87)$ & $<0.05$ \\
\hline Gender $^{\mathrm{b}}$ & & & $>0.05$ \\
\hline Male & $64(65.3)$ & $72(71.3)$ & \\
\hline Female & $34(34.7)$ & $29(28.7)$ & \\
\hline \multicolumn{4}{|l|}{ Etiology $\mathrm{y}^{\mathrm{b}}$} \\
\hline $\mathrm{HBV}$ & 37 (37.7) & $48(47.5)$ & $>0.05$ \\
\hline $\mathrm{HCV}$ & $18(18.3)$ & $10(9.9)$ & $>0.05$ \\
\hline Alcohol & $9(9.2)$ & $4(3.96)$ & $>0.05$ \\
\hline Criptogenic & $25(25.5)$ & $27(26.7)$ & $>0.05$ \\
\hline Mixed & $4(4.0)$ & $11(10.9)$ & $>0.05$ \\
\hline \multicolumn{4}{|l|}{ Child-Pugh class ${ }^{\mathrm{b}}$} \\
\hline A & $13(13.3)$ & $17(16.9)$ & $>0.05$ \\
\hline $\mathrm{B}$ & $37(37.8)$ & $31(30.7)$ & $>0.05$ \\
\hline $\mathrm{C}$ & 48 (48.9) & $53(52.4)$ & $>0.05$ \\
\hline MELD score & $16.18(90)$ & $15.25(92)$ & $>0.05$ \\
\hline Hematocrit $^{\mathrm{a}}$ & $34.32(18.8-53.2)$ & $38.4(20.4-51.6)$ & $<0.001$ \\
\hline Platelet $^{\mathrm{a}}$ & $95(13-1254)$ & $123(25-441)$ & $>0.05$ \\
\hline $\mathrm{INR}^{\mathrm{a}}$ & $1.415(0.92-4.1)$ & $1.32(0.9-6.4)$ & $<0.05$ \\
\hline Albumin $^{\mathrm{a}}$ & $2.97(1.3-5.1)$ & $3.2(1.4-5.2)$ & $<0.05$ \\
\hline Bilirubin $^{a}$ & $2.805(0.56-36.2)$ & $1.6(0.3-42.38)$ & $<0.001$ \\
\hline Aspartate aminotransferase ${ }^{a}$ & $58.5(18-2375)$ & $60(13-3924)$ & $>0.05$ \\
\hline Alanine aminotransferase ${ }^{a}$ & $48(9-1131)$ & $43(8-2420)$ & $>0.05$ \\
\hline Sodium $^{\mathrm{a}}$ & $135(122-143)$ & $137(118-145)$ & $>0.05$ \\
\hline Creatinine $^{\mathrm{a}}$ & $0.775(0.37-5.3)$ & $0.8(0.46-9)$ & $>0.05$ \\
\hline Glucose $^{\mathrm{a}}$ & $119.5(47-552)$ & $98(63-381)$ & $<0.001$ \\
\hline
\end{tabular}

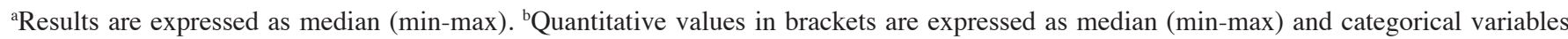
are displayed as percentage. HBV, hepatitis B virus; HCV, hepatitis C virus; PVT, portal vein thrombosis; MELD, Model For End-Stage Liver Disease.

\section{Discussion}

PVT was identified in $10 \%$ of the patients in this study, confirming the increased occurrence of PVT in advanced cirrhosis patients. This result supports those of a previous study indicating that PVT is seen in advanced cirrhosis cases (14). Among the patients in the two groups, hepatitis B virus (HBV) was identified to be the most frequent cause of cirrhosis patients. This result supports a previous study indicating that HBV is the major risk factor for PVT (15).

Different laboratory markers were analyzed in several previous studies $(1,3)$. In the present study, HCT, PLT, INR, ALB, BLB, AST, ALT, Na and CR values were examined. However, only a few of the various laboratory markers studied appeared to affect the risk of developing PVT.

HTC levels were demonstrated to be a risk factor for PVT in the present study. In fact, with splenomegaly, HTC levels decrease as they are affected by hypersplenism and it is already known that hypersplenism is a serious risk factor for PVT $(16,17)$. In a different previous study, the authors demonstrated that the spleen was significantly larger in PVT patients (12). This condition was demonstrated in another
Table II. Prevalence of thrombophilic risk factors in patients with and without portal vein thrombosis.

\begin{tabular}{lccc}
\hline $\begin{array}{l}\text { Thrombophilic } \\
\text { risk factors }\end{array}$ & $\begin{array}{c}\text { PVT } \\
\mathrm{n}(\%)\end{array}$ & $\begin{array}{c}\text { Control } \\
\text { groups n }(\%)\end{array}$ & P-value \\
\hline FVL & $12(12.2)$ & $5(4.9)$ & 0.003 \\
PTHR 20210 & $15(15.3)$ & $11(10.8)$ & 0.18 \\
MTHFR TT677 & $16(16.3)$ & $13(12.8)$ & 0.24 \\
Homocysteine & $17(17.3)$ & $21(20.7)$ & 0.13 \\
Protein C & $73(74.4)$ & $70(69.3)$ & 0.20 \\
Protein S & $71(72.4)$ & $67(66.3)$ & 0.23 \\
Antithrombin III & $63(64.2)$ & $59(58.4)$ & 0.36 \\
\hline
\end{tabular}

FVL, factor V Leiden; PTHR 20210, mutation 20210 of prothrombin gene; MTHFR TT677, the methylenetetrahydrofolate reductase TT677 genotype.

previous study as well (18). A significant association between decreased PLT count and PVT is typically expected, as they 
Table III. The results of backward stepwise multiple logistic regression modeling.

95\% C.I. for OR

\begin{tabular}{lrrrrrr} 
Factors & B & S.E. & P & OR & Lower & Upper \\
\hline Age & 0.020 & 0.011 & 0.059 & 1.020 & 0.999 & 1.042 \\
HTC & -0.102 & 0.025 & 0.000 & 0.903 & 0.860 & 0.949 \\
Constant & 2.496 & 0.991 & 0.012 & 12.134 & &
\end{tabular}

CI, confidence interval; S.E, standard error; OR, Odds ratio. The Hosmer-Lemeshow test indicated that the backward stepwise multiple logistic regression fit well to the data of interest $(\mathrm{P}=0.568)$. The Omnibus tests of model coefficients were statistically significant $(\mathrm{P}<0.001)$.

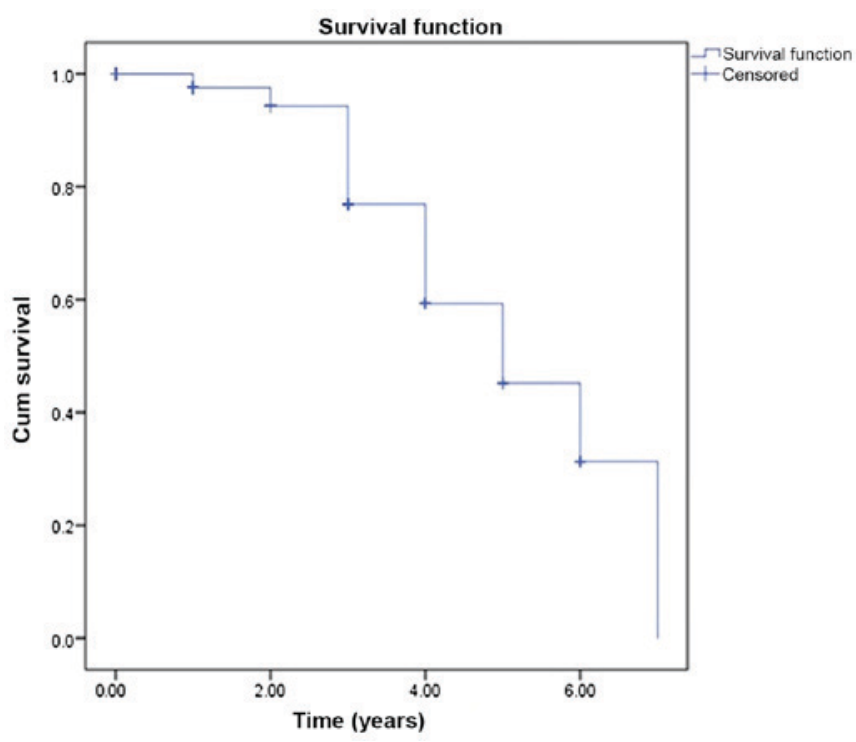

Figure 1. Kaplan-Meier survival curve in patients with portal vein thrombosis according to years. Patient survival was censored at death or loss to follow-up. A small number of patients were lost to follow-up.

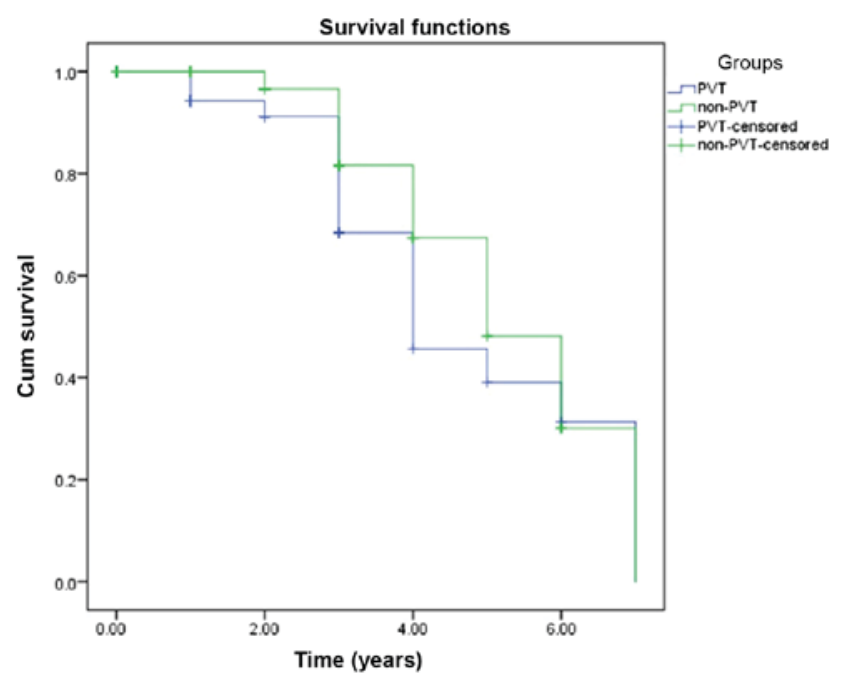

Figure 2. Kaplan-Meier survival curve in patients across groups according to years. PVT, Portal vein thrombosis. Patient survival was censored at death or loss to follow-up. A small number of patients were lost to follow-up. are associated with the same causes in cirrhosis patients. However, in the comparison conducted in this study, no significant differences in PLT were identified between the groups (19-21). The results of other studies support the results of the present study $(7,22)$. In a previous study, it was demonstrated that platelet counts were not associated with venous thromboembolism (VTE) risk of cirrhosis (23).

The liver has a number of hemostatic roles including the production of most coagulation factors and inhibitors, as well as fibrinolytic factors (24). Under normal physiological conditions, there is a stability among procoagulant and anticoagulant factors (25). This delicate hemostatic balance between the two groups works to avoid excessive thrombin generation (3). This stability is disrupted by the effects of advanced liver disease in the form of decreased synthesis of coagulation factors, inhibitors, abnormal clotting factors, abnormalities of fibrinolytic activity, disseminated intravascular coagulation and platelet function defects (24). It is known that reduced levels of the natural anticoagulant antithrombin III (ATIII), protein C (PC) and protein $\mathrm{S}$ (PS) in liver cirrhosis are strong indicators of the disorder of hepatocyte synthetic function. This finding is corroborated further by the positive correlations between the levels of these parameters and other synthetic liver products, including ALB, fibrinogen, and plasminogen in liver cirrhosis (26). The stability between procoagulant and anticoagulant factors is disrupted in association with the severity of liver damage. However, the reduction in natural anticoagulant level is greater compared with other factors (27). In a previous study, the authors demonstrated that plasma levels of anticoagulant proteins, including PC, PS and ATIII, were lower during follow-up in cirrhosis patients who developed PVT compared with those without PVT (24). In a previous retrospective cohort study, the authors demonstrated that a higher INR level did not translate into a decreased risk of thrombosis in cirrhosis patients (28). Therefore, rising INR levels are probably caused by PVT. The results obtained in the present study agree with this hypothesis.

The Child-Turcotte-Pugh (CTP) scoring system is used as a prognostic tool to measure the severity of cirrhosis in patients. In the present study, no significant differences in CTP classes were identified between the groups, which is similar to the results of a previous study (3). Several laboratory markers are used in the CTP scoring system, two of which are BLB and ALB. As such, it is known that increased BLB and low ALB levels parallel the stages of the disease (29). In chronic liver disease, the slowing of portal blood flow associated with the degree of portal hypertension is a condition that creates a tendency toward thrombosis (30). It is well known that portal stream speed is inversely associated with CTP score (31); therefore, the incidence of PVT is increased in cirrhosis patients with CTP class C. In a previous study, the authors demonstrated that chronic liver disease patients with thrombosis had increased BLB levels compared with controls (32). In the present study, elevated BLB levels were associated with a higher incidence of thrombosis in cirrhosis patients. This situation is probably associated with deterioration in portal and hepatic venous stream, which may predispose the patient to PVT. Therefore, BLB and ALB are the two independent risk factors. It is well known that low ALB level may be a reflection of overall hepatic function and reserve. In the current study, serum ALB level was identified to be associated with PVT. 
The associated analyses in the present study suggested that glucose levels may be the only risk factor associated with PVT. Diabetes mellitus, which has previously been considered to be a risk factor for VTE and arterial thrombosis $(33,34)$, may be complicated by microvascular occlusive disease and systemic effects on inflammation, coagulation, and fibrinolysis. This situation has been suggested to cause VTE among patients with diabetes (35). The association between glucose levels and PVT is probably due to the same adverse influences on the vascular wall.

No significant differences in AST or ALT were demonstrated in the comparisons between the groups. The MELD scoring system includes INR, serum BLB and Cr levels. In a previous study, it was demonstrated that a reduction in the level of antithrombotic proteins is strongly associated with the severity of liver cirrhosis in accordance with the MELD system (14). No differences in blood levels of $\mathrm{Na}$ or $\mathrm{Cr}$, which is a member of the MELD, were demonstrated in the comparisons between the groups.

The thrombophilia risk factors that are contained at least a portion (17.6\%) of patients with PVT were effect absolutely on the formation of PVT. The comparison between groups could be performed. It was demonstrated that there was a difference in FVL mutation levels when the groups were compared. These results were demonstrated that was similar to previous studies $(1,36,37)$. Previous studies have demonstrated that certain thrombophilic genotypes, including FVL mutation, may be more frequent in cirrhotic patients with PVT compared with cirrhotic patients without PVT (7).

In a previous study, it was demonstrated that mortality rates were increased in the patients with PVT (11). However, in this study, no differences in survival time were identified in the comparisons between the groups. This situation can explain by the fact that the majority of the patients without PVT in the advanced stage.

As a result, in the present study, it has been demonstrated that patients with hyperglycemia, hypoalbuminemia, anemia, hyperbilirubinemia and elevated INR levels are statistically more likely to have thromboses compared with control group patients. The results of the present study are similar to those of previously published studies of cirrhosis patients $(38,39)$.

The current study had certain limitations as a result of its retrospective design and all the data were obtained from a single center. Therefore, it was a relatively small number of patients. Therefore, larger samples and prospective randomized controlled trials are required to better understand the characteristics of PVT.

Several different risk factors serve a role in the development of PVT in liver cirrhosis and it is important to identify the early development of PVT in cirrhosis patients $(40,41)$. PVT should certainly not be ignored, as it may increase the bleeding risk of varices and cause difficulties associated with liver transplantation in cirrhosis patients $(42,43)$. PVT is mostly asymptomatic, but when patients start to exhibit symptoms the prognosis becomes poor (1). PVT is also associated with increased length of hospital stay and increased costs, leading to an increased health care burden. Therefore, there is a need for parameters to predict PVT. These results remind us that PVT should be considered when evaluating cirrhosis patients. However, treatment against PVT should be considered very cautiously in the absence of absolute contraindications in all these patients. Although these parameters have been studied previously, sample size increases statistical value.

The present study indicates that there is an association between the formation of PVT and several laboratory variables, including HCT, INR, ALB, BLB, glucose and FVL. However, further research efforts are required to better identify and confirm additional risk factors in a larger population.

\section{Acknowledgements}

Not applicable.

\section{Funding}

No funding was received.

\section{Availability of data and materials}

The datasets and materials used during the present study are available from the corresponding author on reasonable request.

\section{Authors' contributions}

YFC conceived and wrote the manuscript. FF and AKA collected the data. CC statistically analyzed the results. YB, IB, OY, MAE, YS and MH designed and interpreted the current study and contributed to further drafts. YFC is the guarantor.

\section{Ethics approval and consent to participate}

Not applicable.

\section{Patient consent for publication}

Not applicable.

\section{Conflict of interest}

The authors declare that they have no conflict of interest regarding the publication of this paper.

\section{References}

1. Amitrano L, Guardascione MA, Brancaccio V, Margaglione M, Manguso F, Iannaccone L, Grandone E and Balzano A: Risk factors and clinical presentation of portal vein thrombosis in patients with liver cirrhosis. J Hepatol 40: 736-741, 2004.

2. Nery F, Chevret S, Condat B, de Raucourt E, Boudaoud L, Rautou PE, Plessier A, Roulot D, Chaffaut C, Bourcier V, et al: Causes and consequences of portal vein thrombosis in 1,243 patients with cirrhosis: Results of a longitudinal study. Hepatology 61: 660-667, 2015.

3. Chen H, Trilok G, Wang F, Qi X, Xiao J and Yang C: A single hospital study on portal vein thrombosis in cirrhotic patients-clinical characteristics \& risk factors. Indian J Med Res 139: 260-266, 2014

4. Fimognari FL and Violi F: Portal vein thrombosis in liver cirrhosis. Intern Emerg Med 3: 213-218, 2008.

5. Ogren M, Bergqvist D, Björck M, Acosta S, Eriksson H and Sternby NH: Portal vein thrombosis: Prevalence, patient characteristics and lifetime risk: A population study based on 23796 consecutive autopsies. World J Gastroenterol 12: 2115-2119, 2006. 
6. Amitrano L, Brancaccio V, Guardascione MA, Margaglione M, Iannaccone L, D'Andrea G, Marmo R, Ames PR and Balzano A: Inherited coagulation disorders in cirrhotic patients with portal vein thrombosis. Hepatology 31: 345-348, 2000

7. Francoz C, Valla D and Durand F: Portal vein thrombosis, cirrhosis, and liver transplantation. J Hepatol 57: 203-212, 2012.

8. Tripodi A, Anstee QM, Sogaard KK, Primignani M and Valla DC: Hypercoagulability in cirrhosis: Causes and consequences. J Thromb Haemost 9: 1713-1723, 2011.

9. Kumar A, Sharma P and Arora A: Review article: Portal vein obstruction-epidemiology, pathogenesis, natural history, prognosis and treatment. Aliment Pharmacol Ther 41: 276-292, 2015

10. Shetty S and Ghosh K: Thrombophilic dimension of Budd chiari syndrome and portal venous thrombosis-a concise review. Thromb Res 127: 505-512, 2011.

11. Englesbe MJ, Kubus J, Muhammad W, Sonnenday CJ, Welling T, Punch JD, Lynch RJ, Marrero JA and Pelletier SJ: Portal vein thrombosis and survival in patients with cirrhosis. Liver Transpl 16: 83-90, 2010

12. Raja K, Jacob M and Asthana S: Portal vein thrombosis in cirrhosis. J Clin Exp Hepatol 4: 320-331, 2014.

13. Durand F and Valla D: Assessment of the prognosis of cirrhosis: Child-Pugh versus MELD. J Hepatol 42 (Suppl 1): S100-S107, 2005.

14. Ponziani FR, Zocco MA, Garcovich M, D'Aversa F, Roccarina D and Gasbarrini A: What we should know about portal vein thrombosis in cirrhotic patients: A changing perspective. World J Gastroenterol 18: 5014-5020, 2012.

15. Lertpipopmetha $\mathrm{K}$ and Auewarakul CU: High incidence of hepatitis B infection-associated cirrhosis and hepatocellular carcinoma in the Southeast Asian patients with portal vein thrombosis. BMC Gastroenterol 11: 66, 2011

16. Kawanaka H, Akahoshi T, Kinjo N, Konishi K, Yoshida D, Anegawa G, Yamaguchi S, Uehara H, Hashimoto N, Tsutsumi N, et al: Impact of antithrombin III concentrates on portal vein thrombosis after splenectomy in patients with liver cirrhosis and hypersplenism. Ann Surg 251: 76-83, 2010.

17. Condat B and Valla D: Nonmalignant portal vein thrombosis in adults. Nat Clin Pract Gastroenterol Hepatol 3: 505-515, 2006.

18. Ushitora Y, Tashiro H, Takahashi S, Amano H, Oshita A Kobayashi T, Chayama K and Ohdan H: Splenectomy in chronic hepatic disorders: Portal vein thrombosis and improvement of liver function. Dig Surg 28: 9-14, 2011.

19. Qi X, Zhang C, Han G, Zhang W, He C, Yin Z, Liu Z, Bai W, Li R, Bai M, et al: Prevalence of the JAK2V617F mutation in Chinese patients with Budd-Chiari syndrome and portal vein thrombosis: A prospective study. J Gastroenterol Hepatol 27: 1036-1043, 2012.

20. Zhang D, Hao J and Yang N: Protein C and D-dimer are related to portal vein thrombosis in patients with liver cirrhosis J Gastroenterol Hepatol 25: 116-121, 2010.

21. Zhang Y, Wen TF, Yan LN, Yang HJ, Deng XF, Li C, Wang C and Liang GL: Preoperative predictors of portal vein thrombosis after splenectomy with periesophagogastric devascularization. World J Gastroenterol 18: 1834-1839, 2012.

22. Lisman T and Porte RJ: The role of platelets in liver inflammation and regeneration. Semin Thromb Hemost 36: 170-174, 2010.

23. Northup PG, McMahon MM, Ruhl AP, Altschuler SE, Volk-Bednarz A, Caldwell SH and Berg CL: Coagulopathy does not fully protect hospitalized cirrhosis patients from peripheral venous thromboembolism. Am J Gastroenterol 101: 1524-1528, 2006.

24. Zocco MA, Di Stasio E, De Cristofaro R, Novi M, Ainora ME, Ponziani F, Riccardi L, Lancellotti S, Santoliquido A, Flore R, et al: Thrombotic risk factors in patients with liver cirrhosis: Correlation with MELD scoring system and portal vein thrombosis development. J Hepatol 51: 682-689, 2009.
25. Roberts LN, Patel RK and Arya R: Haemostasis and thrombosis in liver disease. Br J Haematol 148: 507-521, 2010.

26. Al Ghumlas AK, Abdel Gader AG and Al Faleh FZ: Haemostatic abnormalities in liver disease: Could some haemostatic tests be useful as liver function tests? Blood Coagul Fibrinolysis 16: 329-335, 2005

27. Vukovich T, Teufelsbauer H, Fritzer M, Kreuzer S and Knoflach P: Hemostasis activation in patients with liver cirrhosis. Thromb Res 77: 271-278, 1995.

28. Dabbagh O, Oza A, Prakash S, Sunna R and Saettele TM Coagulopathy does not protect against venous thromboembolism in hospitalized patients with chronic liver disease. Chest 137: 1145-1149, 2010.

29. Nicoll A: Surgical risk in patients with cirrhosis. J Gastroenterol Hepatol 27: 1569-1575, 2012

30. Fimognari FL, De Santis A, Piccheri C, Moscatelli R, Gigliotti F, Vestri A, Attili A and Violi F: Evaluation of D-dimer and factor VIII in cirrhotic patients with asymptomatic portal venous thrombosis. J Lab Clin Med 146: 238-243, 2005.

31. Zironi G, Gaiani S, Fenyves D, Rigamonti A, Bolondi L and Barbara L: Value of measurement of mean portal flow velocity by Doppler flowmetry in the diagnosis of portal hypertension. J Hepatol 16: 298-303, 1992.

32. Anthony Lizarraga W, Dalia S, Reinert SE and Schiffman FJ: Venous thrombosis in patients with chronic liver disease. Blood Coagul Fibrinolysis 21: 431-435, 2010.

33. Piazza G, Goldhaber SZ, Kroll A, Goldberg RJ, Emery C and Spencer FA: Venous thromboembolism in patients with diabetes mellitus. Am J Med 125: 709-716, 2012.

34. Movahed MR, Hashemzadeh M and Jamal MM: The prevalence of pulmonary embolism and pulmonary hypertension in patients with type II diabetes mellitus. Chest 128: 3568-3571, 2005.

35. Lowe GD: Common risk factors for both arterial and venous thrombosis. Br J Haematol 140: 488-495, 2008

36. Egesel T, Büyükasik Y, Dündar SV, Gürgey A, Kirazli S and Bayraktar Y: The role of natural anticoagulant deficiencies and factor $\mathrm{V}$ Leiden in the development of idiopathic portal vein thrombosis. J Clin Gastroenterol 30: 66-71, 2000.

37. Qi X, Ren W, De Stefano V and Fan D: Associations of coagulation factor V Leiden and prothrombin G20210A mutations with Budd-Chiari syndrome and portal vein thrombosis: A systematic review and meta-analysis. Clin Gastroenterol Hepatol 12: 1801-1812.e7, 2014

38. Weber A, Krebs S, Lenhardt C, Wagenpfeil S, Schmid RM and Schulte-Frohlinde E: Correlation of routinely used coagulation parameters and presence of portal vein thrombosis in patients with liver cirrhosis. Hepatol Res 39: 882-887, 2009.

39. Chen H, Qi X, He C, Yin Z, Fan D and Han G: Coagulation imbalance may not contribute to the development of portal vein thrombosis in patients with cirrhosis. Thromb Res 131: 173-177, 2013.

40. Mantaka A, Augoustaki A, Kouroumalis EA and Samonakis DN: Portal vein thrombosis in cirrhosis: Diagnosis, natural history, and therapeutic challenges. Ann Gastroenterol 31: 315-329, 2018.

41. Denninger MH, Chaït Y, Casadevall N, Hillaire S, Guillin MC, Bezeaud A, Erlinger S, Briere J and Valla D: Cause of portal or hepatic venous thrombosis in adults: The role of multiple concurrent factors. Hepatology 31: 587-591, 2000.

42. Stine JG, Pelletier SJ, Schmitt TM, Porte RJ and Northup PG: Pre-transplant portal vein thrombosis is an independent risk factor for graft loss due to hepatic artery thrombosis in liver transplant recipients. HPB (Oxford) 18: 279-286, 2016.

43. Englesbe MJ, Schaubel DE, Cai S, Guidinger MK and Merion RM: Portal vein thrombosis and liver transplant survival benefit. Liver Transpl 16: 999-1005, 2010. 